\title{
CONSERVAÇÃO E PROTEÇÃO DA ÁGUA SUBTERRÂNEA: UMA REVISÃO DE METODOLOGIAS DE MAPEAMENTO DE AQUÍFEROS PARA O ORDENAMENTO TERRITORIAL
}

\author{
Filipe da Silva Peixoto \\ Universidade do Estado do Rio Grande do Norte, Departamento de Geografia \\ felipepeixoto@uern.br \\ Itabaraci Nazareno Cavalcante \\ Universidade Federal do Ceará, Departamento de Geologia \\ itabaracicavalcante@gmail.br \\ Karen Vendramini de Araújo \\ Universidade Federal do Ceará, Departamento de Geologia \\ karenvendramini@yahoo.com.br
}

Renata Nayara Miranda Câmara Silveira Universidade Federal do Ceará, Departamento de Geologia renayarac@gmail.com

\begin{abstract}
RESUMO
O uso sustentável dos recursos hídricos é uma necessidade para garantir o direito do acesso à água para futuras gerações. No Brasil, apesar dos entes estaduais e união, serem os responsáveis pela gestão dos recursos hídricos, a municipalidade possui o papel de desenvolver sistemas racionais de ordenamento territorial para minimizar os efeitos do uso e ocupação do solo sobre a água. Para isso é necessário o auxílio de metodologias de vulnerabilidade aquífera, haja vista importância estratégica da água subterrânea para o desenvolvimento socioeconômico e sustentável do território. O referido trabalho faz uma sistematização das metodologias de aplicação da vulnerabilidade aquífera, avançando na construção desse conceito para o ordenamento territorial em abordagem interdisciplinar para conservação e proteção dos recursos hídricos subterrâneos. A vulnerabilidade aquífera e o risco associado à ação da sociedade, devem compor o diagnóstico ambiental que gera subsídios para indicar áreas de diferentes graus de risco à contaminação, em função do tipo de aquífero e atividades potencialmente geradoras de contaminantes.
\end{abstract}

Palavras-chave: Recursos hídricos. Contaminação hídrica. Caracterização aquífera. Planejamento do território.

\section{CONSERVATION AND PROTECTION OF THE GROUNDWATER: METHODOLOGICAL REVIEW OF THE AQUIFER MAPPING FOR TERRITORIAL ORDERING}

\begin{abstract}
Sustainable use of the water resource is a necessity for promote access to water for future generation. In Brazil, the stats and union, in some cases, are responsible for water resource management, while the municipality has been responsible for territorial ordering. For this, is necessary the auxiliary of aquifer vulnerability methodologies, since the strategic importance of water on socioeconomic development and territorial sustainability. This work arms to systematize the application methodologies of aquifer vulnerability, advancing in construction this concept, and addressing it to geographic and interdisciplinary approach applied to research in conservation and preservation of groundwater resources, using territorial planning as a tool. The vulnerability of the aquifer and the risk associated with anthropogenic interference, promoting the environmental diagnosis that generates subsidies to indicate different degrees of the hazard to contamination, according to the activity with contaminate potential.
\end{abstract}

Keywords: Water resource. Water pollution. Aquifer characterization. Territorial planning. 


\section{INTRODUÇÃO}

$\mathrm{Na}$ atualidade, cada vez mais as condições de desenvolvimento econômico dependem da forma de equacionamento dos recursos naturais. Com uma grande demanda de recursos para o abastecimento urbano e atividades produtivas, a água é um insumo chave para viabilizar, também, a produção agrícola e industrial (CHEN et al., 2014; ROGERS, 2006). A água possui reconhecidamente uma quantidade constante no globo, contudo, a diminuição e mesmo esgotamento de reservatórios e perda da qualidade hídrica podem elevar a custos locacionais e operacionais e, consequentemente, causar maior escassez e menos acesso a esse recurso (OKE e KANAl, 2006).

A água é dotada de valor econômico, e sua característica de uso múltiplo envolve diversos setores da sociedade, a exemplo dos diferentes setores agrícolas, industriais e abastecimento doméstico. Portanto, esse recurso estratégico é passível de ser a causa de diversos conflitos entre usuários e até mesmo entre nações (MORAG, 2010; SWAIN, 2011).

O Estado moderno, como um mediador entre interesses de diferentes grupos sociais, tem o papel de gerir a água de maneira a equacionar sua relativa condição de escassez com a partição justa e equilibrada desse recurso. Contudo, fica claro que pela relação entre as condições qualitativas e quantitativas dos recursos hídrico, a gestão vai para além da alocação. Para Campos (2001, p. 23) a gestão das águas é "o conjunto de procedimentos organizados no sentido de solucionar os problemas referentes ao uso e ao controle dos recursos hídricos". Mas envolve também a relação sociedade-natureza, à medida que a construção social dos espaços pode impactar de diferentes modos no meio ambiente, e com isso também na condição de qualidade e quantidade da água no reservatório (TUNDISI, 2008; PEIXOTO, 2017).

O meio subterrâneo possui a parcela da água na terra mais passível de ser explotada. Contudo, ainda não se encontram condições efetivas e eficientes de gerenciamento desse recurso. Isso se deve à falta de investimentos em pesquisas de quantificação da água subterrânea em reservatórios e deficiências desenvolvimento de estratégias de gerenciamento e planejamento hídrico integrado entre a água superficial e subterrânea (REBOUÇAS, 1997; JARVIS, 2005). Nos países em desenvolvimento como o Brasil, a água subterrânea é bastante utilizada como fonte de abastecimento. Villar (2016) confere a grande importância desta para pequenos municípios, sobretudo nas zonas rurais onde abastece $55,5 \%$ dos municípios do Brasil.

Os reservatórios de água subterrânea são aquíferos, representadas pelas formações geológicas que armazenam quantidades relativamente significativas de água e são capazes de cedê-la, portanto, são propícios à explotação por meio de poços ou fontes (FETTER ,1994; FREEZE e CHERRY, 1987). Por se encontrar no subsolo, a água subterrânea possui maior proteção à poluição e à contaminação do que nos reservatórios superficiais (FOSTER et al., 2007, JANG et al, 2015). Contudo, a contaminação de um aquífero pode resultar na inviabilidade do uso do seu recurso hídrico durante décadas, além de custos e tempo excessivamente maiores para a descontaminação.

Medidas preventivas são as mais eficientes para a conservação dos aquíferos e das águas subterrâneas. Identificar áreas potencialmente mais vulneráveis é essencial para o planejamento territorial. A efetividade e qualidade desse tipo de zoneamento se dá em função de subsídios resultantes de pesquisas científicas que promovam estratégias para o gerenciamento hídrico ambiental e territorial integrados (MURAT, 2000; LOTIF, 2014).

As propriedades distintas dos aquíferos os classificam em tipos, no que tange a potencial contaminação e envolvem, sobretudo, parâmetros que indicam mais ou menos acessibilidade, ou entrada de contaminantes. Com essa temática, a vulnerabilidade aquífera se refere às características, propriedades e atributos naturais do aquífero quanto à resistência que este oferece ao acesso do contaminante à água subterrânea.

A vulnerabilidade aquífera, desse modo, pode ser determinada a partir de parâmetros intrínsecos ao aquífero como: nível estático, condutividade hidráulica, transmissividade, grau de confinamento ou não confinamento do aquífero, tipo de aquífero (meio cárstico, intersticial ou fissural), tipo de litologia e grau de fraturamento das rochas (CIVITA, 1990; AUBRE, 1992; EPA, 1993; VRBA e ZAPOROZEC, 1994), ou ainda, atributos naturais extrínsecos ao aquífero como: tipo de solo, topografia do terreno, e quantificação da recarga (ALLER, 1987; JANG e CHEN, 2015; MUHAMMAD et al., 2015).

A recarga do aquífero é um parâmetro que envolve tanto a condição quantitativa como qualitativa. Além da recarga depender direta ou indiretamente da pluviosidade, pode transferir materiais contaminantes 
dispostos de maneira difusa, pontual ou linear na superfície do solo ou na zona subsaturada, seja de forma natural ou induzida, intencional ou não intencionalmente pelo homem.

A preocupação de ordenamento do território, de modo a garantir a disponibilidade de recursos para desenvolvimento econômico e garantir o abastecimento urbano, foi o motivo para os primeiros estudos cartográficos a respeito da proteção dos aquíferos. Albinet e Margat (1970) realizaram o mapeamento da vulnerabilidade aquífera no território francês em uma escala de 1:1.000.000 em função da profundidade da zona subsaturada e de fatores que influenciam na quantidade de recarga anual.

A evolução dessa abordagem leva ao desenvolvimento do conceito de vulnerabilidade à contaminação nos aquíferos. De acordo com as conclusões da Internacional Confference Vulnerability of Soil and Groundwater to Pollutants realizada em 1987, a vulnerabilidade aquífera é definida como a sensibilidade da qualidade das águas subterrâneas às cargas poluentes. Ou seja, em função, apenas, das características intrínsecas do aquífero.

Outro conceito importante nesse contexto é o de risco. Este se refere às atividades humanas que são potencialmente contaminantes/poluentes sobre os aquíferos e águas subterrâneas. A combinação entre a vulnerabilidade e o risco resulta no grau de perigo. O grau de perigo é entendido e consagrado nas publicações internacionais como "hazard" se refere diretamente à probabilidade de contaminação e/ou poluição.

Kazakis e Voudouris (2015); respectivamente, definem vulnerabilidade das águas subterrâneas como: vulnerabilidade intrínseca que se refere às características naturais dos aquíferos, refletindo seus parâmetros hidrodinâmicos e potencial de depuração de contaminantes. Gogu e Dassargues, (2000) se referem a vulnerabilidade específica quanto ao comportamento de inserção, propagação e persistência de certo tipo de contaminante ou grupo de contaminantes com caraterísticas similares.

O artigo, ora apresentado, busca sistematizar as metodologias de aplicação da vulnerabilidade aquífera, avançando na construção desse conceito como ferramenta efetiva de aplicação para o ordenamento territorial.

\section{METODOLOGIA}

O referido trabalho foi pautado em pesquisa bibliográfica, realizada de forma sistemática, de modo a articular as produções científicas mais relevantes relacionadas à vulnerabilidade aquífera. O levantamento ocorreu com ênfase em artigos publicados nos últimos 18 anos, em periódicos científicos internacionais indexados, haja vista que, foi buscado mostrar a evolução e tendências das metodologias de vulnerabilidade aquífera e trabalhos clássicos que também foram publicados em teses, e relatórios técnicos.

O levantamento foi realizado por meio de pesquisa exaustiva com o método "bola de neve" quando os manuscritos começam a se repetir ciclicamente, ocorrendo assim articulação significativa entre a produção científica sobre o tema e os dados bibliográficos que compõem o artigo. De acordo com Vinutu (2014), apesar das limitações desse tipo de amostragem, ele é útil para pesquisas em grupos temáticos elementos pouco expressivos.

\section{RESULTADOS E DISCUSSÃO}

Existe uma multiplicidade de metodologias de aplicados a conservação e proteção das águas subterrâneas. As primeiras, desenvolvidas na França na década de 70 foram aplicadas como ferramentas para o ordenamento territorial pelo Governo francês, apontando locais menos vulneráveis para determinadas atividades econômicas ou de ocupação.

\section{O Ordenamento territorial para a proteção e conservação das águas subterrâneas}

O termo ordenamento territorial criou forma no fundamento positivista de colocar "ordem", nas condições de desordem material, amparando as arestas formadas pela economia de mercado e externalidades do capital. No contexto pós-segunda guerra o Aménagement Territoire na França, assim como o Regional Planning no EUA tornaram-se sinônimos do ordenamento territorial. Esses têm sido aplicados como

\begin{tabular}{|c|c|}
\hline & Uberlândia-MG \\
\hline
\end{tabular}


instrumentos que possuem a função de valorizar, explorar e desenvolver o território de um modo organizado (PEREZ e CHIQUITO, 2012).

Estaba (1999) foca as ações de ordenamento abordando as potencialidades de territórios para a expansão e aumento de sua competitividade sistêmica, além da superação da oposição entre desenvolvimento e conservação ambiental. A abordagem do ordenamento territorial, para além da aplicação de planos, também aplica-se no controle do uso do solo por meio de medidas de restrição, e isso se percebe no Spatial Planning utilizado na Inglaterra, e na implantação de Master Plans ou Planos Diretores para o ordenamento de grandes cidades da França, Itália e Grécia (ARAÚJO, 2014). Essa estratégia foi implantada também no Brasil, onde o instrumento do Plano Diretor é usado como articulador da política urbana brasileira baseada no Estatuto das Cidades sob a Lei no 10.257/2001 (PEIXOTO, 2017).

Araújo (2014) postula que o ordenamento territorial passou de uma abordagem estruturalista para pósestruturalista. Há, portanto, a transição de uma abordagem racional concentrada no Estado Moderno para uma abordagem participativa, onde a sociedade tem o direito e dever de participar do planejamento e de tomadas de decisões. Contudo, a necessidade de subsídios técnicos para dar suporte às decisões vem crescendo com o aumento das demandas de espaço para atividades produtivas, serviços e assentamentos urbanos, sendo essencial para isso a efetivação da política ambiental.

O zoneamento, principalmente em áreas urbanas, tem se mostrado útil nas políticas de ordenamento territorial no Brasil. Segundo Villaça (1999) e Rezende (2006) o zoneamento as leis de zoneamento e loteamentos têm tido uma existência real e têm sido objeto do teste da prática.

O ordenamento territorial possui instrumentos que abordam diferentes perspectivas de aplicação. Erkan (2008) os define como:

- Planejamento estratégico de longo prazo - utiliza a síntese de uma visão de onde se quer chegar (objetivos) e após a aplicação, ocorre avaliação para correção e remodelamento estratégica das ações. Esse processo é conhecido como evaluation of strengths, weaknesses, opportunities and threats-SWOT.

- Planos - trata-se do desenvolvimento em diferentes escalas geográficas, incluindo nacional, regional, urbana ou em recorte de bairros. Os planos incluem estratégias, políticas, projetos temáticos como: habitação; turismo qualidade ambiental etc.

- Desenvolvimento de controle de locação - utiliza zoneamentos e restrições uso do solo, bem como restrições de tipos de construções operando no nível local.

Entende-se, segundo Friedmain (2005), que o processo de ordenamento se refere a suportar a melhor qualidade da vida humana por meio da equidade e justiça social, além de aspectos estéticos e ambientais. A conservação e planejamento estratégico dos recursos hídricos são necessariamente incluídos no ordenamento do território, pois a importância desse recurso para a economia, desenvolvimento social e conservação ambiental exigem níveis cada vez articulados de políticas de gestão das águas e gestão do território, além de prestar informações mais precisas acerca da totalidade do objeto estudado.

As pesquisas de vulnerabilidade aquífera são essenciais nesse contexto, pois a água subterrânea é um recurso chave, sobretudo, por causa das diferenciações das propriedades hidrogeológicas e hidrodinâmicas dos aquíferos. Assim, é necessário identificar quais deles possuem maior vulnerabilidade à contaminação das águas subterrâneas em função do uso do solo. Este tipo de zoneamento permite estabelecer zonas, em variados graus de proteção, essenciais para a conservação dos aquíferos.

Tal perspectiva de aplicação pode definir ou compor mapas sintéticos de potencialidade e limitações do uso do solo. E, ainda, mapas de formas integradas com os sistemas ambientais classificados a partir da análise da paisagem. 


\section{Vulnerabilidade aquífera e riscos potenciais}

De acordo com a National Resource Courcil (1993) têm-se que: i) todo aquífero possui algum grau de vulnerabilidade; ii) incertezas são inerentes a toda avaliação de vulnerabilidade; iii) em estado de vulnerabilidade aquífera existe a possibilidade de que o óbvio esteja obscurecido, seja pela complexidade do aquífero seja pela aplicação de métodos inadequados.

A dificuldade de representação de estudos de vulnerabilidade aquífera é causada pela diversidade dos aquíferos e dificuldade de investigá-los, além da tentativa de aplicação das metodologias em várias áreas sem as adaptações necessárias. Assim, a avaliação relativa é mais comum e estas são importantes para o ordenamento territorial (HIRATA, 2001; MORAIS, 2016). Para Lubianetzky et al. (2015), as metodologias intrínsecas de vulnerabilidade aquífera estão proporcionalmente relacionadas à quantidade de recarga do aquífero por infiltração através da zona sub-saturada atravessando ou preenchendo poros ou fraturas.

O termo vulnerabilidade remonta a década de 1960 e foi escolhido a fim de sensibilizar a população de que mesmo a água subterrânea sendo associada à pureza, ela está passível de sofrer poluição e/ou contaminação por agentes externos (MURAT, 2000). Os primeiros estudos para cartografar a susceptibilidade dos aquíferos se basearam na sobreposição de informações de nível estático de poços, informações estratigráficas e fontes potenciais de poluição, para definir estratégias de conservação das águas subterrâneas na França (ALBINET e MARGAT, 1970). Os autores, ainda consideram, para isso, 03 princípios básicos: a) Entrada de poluentes no sistema - introdução de fluidos por meio da parte superficial do solo, quando o aquífero é livre; b) A propagação - dependente das condições de movimento das águas subterrâneas, refletidas pelo grau de permeabilidade do meio aquífero, propriedades hidrodinâmicas de condutividade hidráulica e transmissividade são parâmetros que permitem mensurar essa condição; c) Persistência do poluente - persistência deste no aquífero por dezenas e até centenas de anos, mesmo que cessada a fonte contaminante.

Assim, a vulnerabilidade aquífera continua sendo o principal fundamento para as metodologias aplicadas a conservação e proteção dos recursos hídricos subterrâneos. Vrba e Zaporozec (1994) as classificaram em 03 distintos grupos: a) Ambientes hidrogeológicos: baseiam a avaliação da vulnerabilidade, em termos qualitativos, nas características gerais do ambiente, usando mapas temáticos (ALBINET e MARGAT, 1970); b) Modelos análogos: utilizam expressões matemáticas para os parâmetros essenciais, tais como, tempo de trânsito médio na zona vadosa como indicadores do índice de vulnerabilidade (MONKHOUSE, 1983); c) Sistemas paramétricos: usam parâmetros selecionados como indicadores de vulnerabilidade e aplicam seu espectro de valores e interações para produzir alguma forma de índice de vulnerabilidade relativo ou absoluto. Inclui como exemplo desse, o consagrado método DRASTIC de Aller et al. (1987).

Outros tipos de classificação descritos por Evans e Maidment (1995) colocam que há 03 tipos de modelos que agrupam as metodologias de vulnerabilidade aquífera: (1) Métodos baseados em índices paramétricos - estes utilizam pesos para diferentes indicadores gerando mapas sintéticos; (2) métodos empregando modelos de simulação - nestes são aplicados fundamentos físicos e matemáticos de interação entre o meio, a água e o contaminante, identificando o seu comportamento na água subterrânea; (3) modelos estatísticos usados para testar outros métodos e identificar variáveis mais importantes, e em seguida, testar a eficiência do método, por meio de análises geoestatísticas calibradas por amostras de campo servindo, portanto, de base para modificações de métodos paramétricos ou de simulação.

Kumar et al. (2015) similarmente, entendem que os modelos de mapeamento da vulnerabilidade aquífera são divididos em: modelos estatísticos, processos baseados em simulação matemática e modelos baseados em índices. Estes últimos podem ser divididos em paramétrico, não paramétrico e híbrido (Figura 1). Os métodos paramétricos são mais utilizados, de modo que, não incubem aos modelos computacionais a totalidade das decisões dos níveis de vulnerabilidade criados e, assim, dão mais autonomia ao pesquisador.

Há uma evolução perceptível nas metodologias de vulnerabilidade aquífera e estas vêm sendo adaptadas a partir de aplicações mais gerais para aplicações específicas, sendo criadas metodologias mais aplicadas a diferentes tipos de aquíferos e resultados cartográficos de vulnerabilidade e risco associados localmente, enquanto os modelos estatísticos também têm sido desenvolvidos, porém de maneira menos intensa que os métodos paramétricos (KUMAR et al., 2015; BRINDA e ELANGO, 2015) (Quadro 1):

\begin{tabular}{llllll}
\hline Caminhos de Geografia & Uberlândia-MG & v. 21, n. 75 & Jun/2020 & p. 01-14 Página 5
\end{tabular}


Conservação e proteção da água subterrânea: uma revisão de metodologias de mapeamento de aquíferos para o ordenamento territorial
Filipe da Silva Peixoto

Itabaraci Nazareno Cavalcante

Karen Vendramini de Araújo Renata Nayara Miranda Câmara Silveira

Figura 1 - Modelo esquemático de tipos de vulnerabilidade aquífera.

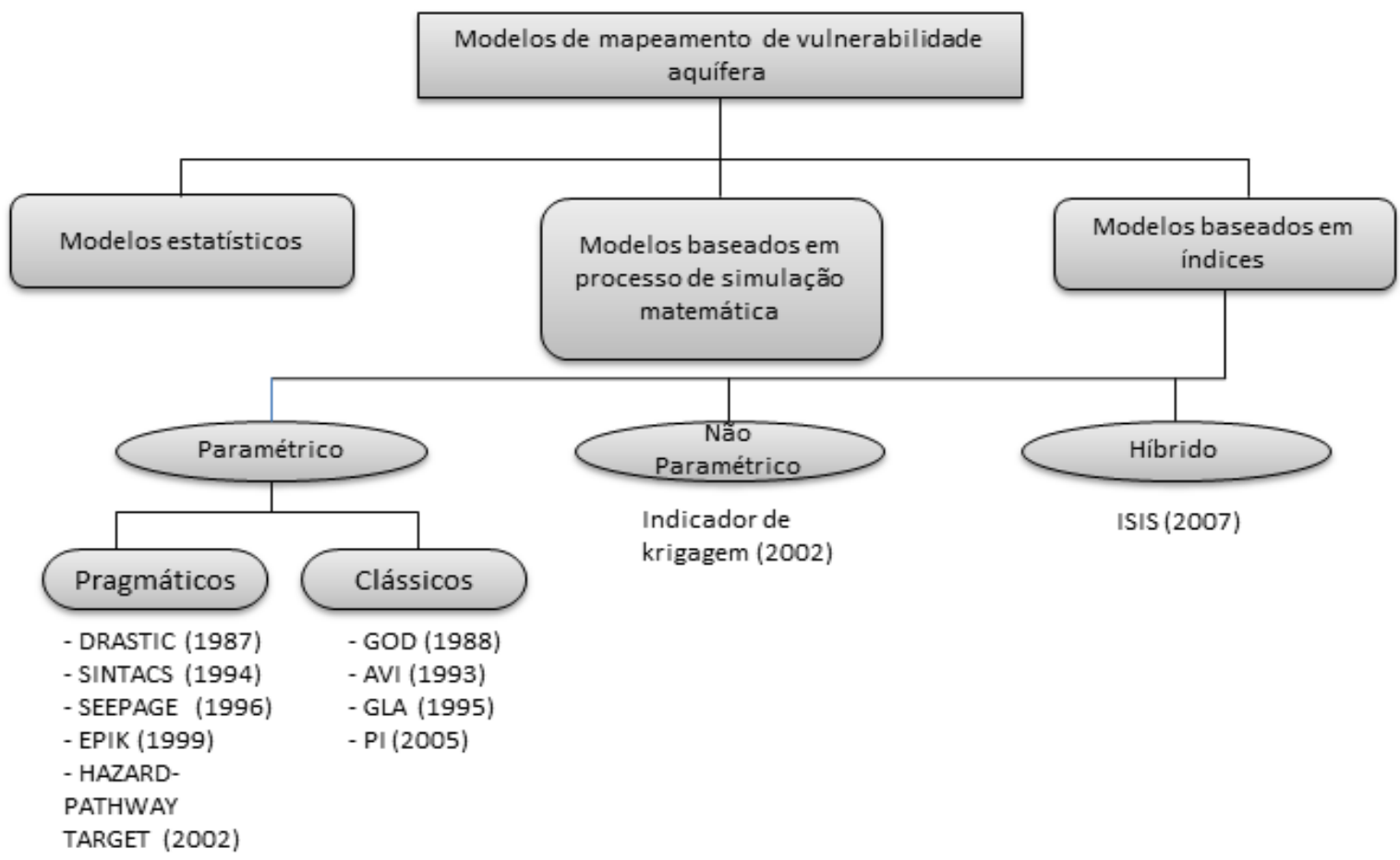

Fonte: Adaptado de Kumar et al., (2015).

Quadro 1 - Principais metodologias de vulnerabilidade aquífera.

\begin{tabular}{|c|c|c|c|}
\hline Referência & $\begin{array}{c}\text { Nome do } \\
\text { método }\end{array}$ & Variáveis utilizadas & Aplicações \\
\hline Le Grand (1964) & $\begin{array}{c}\text { Surface } \\
\text { impoundment } \\
\text { Assessment }\end{array}$ & $\begin{array}{l}\text { Profundidade da zona subsaturada; } \\
\text { Recarga; Qualidade da água; } \\
\text { Periculosidade do material }\end{array}$ & $\begin{array}{c}\text { Avaliação da água } \\
\text { subterrânea para o uso }\end{array}$ \\
\hline Hargerty (1973) & $\begin{array}{l}\text { Site Ranking } \\
\text { System }\end{array}$ & $\begin{array}{l}\text { Solo; condutividade hidráulica; sorção; } \\
\text { tamponamento químico; hidrodinâmica; } \\
\text { população }\end{array}$ & $\begin{array}{l}\text { Análise de disposição de } \\
\text { produtos químicos }\end{array}$ \\
\hline Taltasse (1972) & Sem nome & Geologia (litologia; estrutura) & Vulnerabilidade aquífera \\
\hline $\begin{array}{l}\text { Philips et al. } \\
\text { (1977) }\end{array}$ & $\begin{array}{l}\text { Waste-soil } \\
\text { Interaction } \\
\quad \text { Matrix }\end{array}$ & $\begin{array}{c}\text { Efeitos na saúde; característica do } \\
\text { produto químico; Comportamento do } \\
\text { produto; Capacidade de atenuação do } \\
\text { solo; Hidrogeologia; Características do } \\
\text { local } \\
\end{array}$ & $\begin{array}{l}\text { Análise de disposição de } \\
\text { resíduos sólidos e } \\
\text { líquidos e novas } \\
\text { indústrias; Vulnerabilidade } \\
\text { específica } \\
\end{array}$ \\
\hline Kulfs (1980) & $\begin{array}{l}\text { Site Ranking } \\
\text { Methodology }\end{array}$ & $\begin{array}{l}\text { População; Uso da água; Uso local; } \\
\text { Degradação ambiental; Contaminação; } \\
\text { Profundidade do nível d'água; } \\
\text { Pluviometria; Permeabilidade; } \\
\text { Característica do resíduo }\end{array}$ & $\begin{array}{l}\text { Análise de disposição de } \\
\text { resíduos sólidos e } \\
\text { líquidos e novas } \\
\text { indústrias; Vulnerabilidade } \\
\text { específica }\end{array}$ \\
\hline $\begin{array}{l}\text { Silva et al. } \\
\quad(1980)\end{array}$ & Tpe & $\begin{array}{l}\text { Velocidade da água subterrânea; } \\
\text { Porcentagem de argila; Atividade } \\
\text { potencialmente contaminante; } \\
\text { Explotação dos aquíferos }\end{array}$ & $\begin{array}{l}\text { Áreas de perigo à } \\
\text { contaminação }\end{array}$ \\
\hline Duarte (1980) & $\begin{array}{c}\text { Mapa de } \\
\text { vulnerabilidade }\end{array}$ & Litologia; Área de recarga e descarga & Vulnerabilidade geral \\
\hline $\begin{array}{l}\text { Caldwell et al. } \\
\quad(1981)\end{array}$ & $\begin{array}{l}\text { Hazard Ranking } \\
\text { System }\end{array}$ & $\begin{array}{l}\text { Migração-característica do meio e } \\
\text { resíduo; Quantidade de produto; } \\
\text { População próxima; Explosão e fogo; } \\
\text { Contato direto }\end{array}$ & $\begin{array}{l}\text { Áreas prioritárias para } \\
\text { limpeza do aquífero }\end{array}$ \\
\hline Western & Brine Disposal & Método de disposição; Volume; & Avaliação de águas de \\
\hline
\end{tabular}


Conservação e proteção da água subterrânea: uma revisão de metodologias de mapeamento de aquíferos para o ordenamento territorial
Filipe da Silva Peixoto

Itabaraci Nazareno Cavalcante

Karen Vendramini de Araújo Renata Nayara Miranda Câmara Silveira

\begin{tabular}{|c|c|c|c|}
\hline $\begin{array}{c}\text { Michigan } \\
\text { University } \\
(1981)\end{array}$ & Methodology & $\begin{array}{c}\text { Geologia; Densidade de poços de } \\
\text { petróleo; Proximidade dos poços de água }\end{array}$ & $\begin{array}{l}\text { formação em campos de } \\
\text { petróleo e gás; } \\
\text { Vulnerabilidade específica }\end{array}$ \\
\hline Le Grand (1983) & $\begin{array}{l}\text { Landfill Site } \\
\text { Ranking } \\
\text { (Método Le } \\
\text { Grand-Brown) }\end{array}$ & $\begin{array}{c}\text { Distância aterro e poços produtores; } \\
\text { Profundidade do nível d'água; Gradiente } \\
\text { do aquífero; Permeabilidade; Capacidade } \\
\text { de atenuação }\end{array}$ & $\begin{array}{c}\text { Avaliação de aterros } \\
\text { sanitários novos e em } \\
\text { operação; Vulnerabilidade } \\
\text { específica }\end{array}$ \\
\hline Haertle (1983) & (sem nome) & $\begin{array}{l}\text { Composição do solo; Espessura da } \\
\text { camada não-saturada. }\end{array}$ & $\begin{array}{l}\text { Vulnerabilidade de } \\
\text { aquíferos não-confinados }\end{array}$ \\
\hline Rao et al. (1985) & Pesticide Index & $\begin{array}{c}\text { Característica físico-química do } \\
\text { pesticida; Clima; Perfil do solo; Cultura }\end{array}$ & $\begin{array}{l}\text { Avaliação de pesticidas, } \\
\quad \text { uso normal; } \\
\text { Vulnerabilidade específica }\end{array}$ \\
\hline Aller (1987) & DRASTIC & $\begin{array}{l}\text { Profundidade da zona subsaturada; } \\
\text { recarga; meio aquífero; topografia; } \\
\text { impacto da zona vadosa; condutividade } \\
\text { hidráulica }\end{array}$ & Vulnerabilidade aquífera \\
\hline $\begin{array}{l}\text { Carter et al. } \\
\quad(1987)\end{array}$ & $\begin{array}{l}\text { Groundwater } \\
\text { Vulnerability } \\
\text { Map for Nitrate }\end{array}$ & $\begin{array}{l}\text { Tipo de solo; Características hidráulicas; } \\
\text { Litologia }\end{array}$ & $\begin{array}{l}\text { Avaliação do potencial de } \\
\text { lixiviação de nitrato; } \\
\text { Vulnerabilidade à } \\
\text { contaminante específico }\end{array}$ \\
\hline $\begin{array}{l}\text { Marcolongo \& } \\
\text { Pretto (1987) }\end{array}$ & (sem nome) & $\begin{array}{l}\text { Condutividade hidráulica da zona não } \\
\text { saturada; Profundidade da água } \\
\text { subterrânea; Umidade do solo; Recarga } \\
\text { real. }\end{array}$ & $\begin{array}{l}\text { Vulnerabilidade geral, } \\
\text { baseado na teoria do } \\
\text { fluxo-pistão }\end{array}$ \\
\hline $\begin{array}{l}\text { Foster \& Hirata } \\
\text { (1988) }\end{array}$ & GOD & $\begin{array}{c}\text { Tipo de aquífero; litologia da zona } \\
\text { vadosa; profundidade da zona } \\
\text { subsaturada }\end{array}$ & Vulnerabilidade aquífera \\
\hline $\begin{array}{l}\text { Civita et al., } \\
\text { (1990) }\end{array}$ & SINTACS & $\begin{array}{l}\text { Profundidade da água subterrânea; tipo } \\
\text { de solo; infiltração; aquífero; litologia da } \\
\text { zona subsaturada; condutividade; } \\
\text { topografia }\end{array}$ & Vulnerabilidade aquífera \\
\hline $\begin{array}{c}\text { Adams \& Foster } \\
(1992)\end{array}$ & Sem nome & $\begin{array}{l}\text { Caracteristica litológica; permeabilidade; } \\
\text { profundidade da zona subsaturada }\end{array}$ & Vulnerabilidade aquífera \\
\hline $\begin{array}{l}\text { Foster \& Hirata } \\
\text { (1993) }\end{array}$ & $\begin{array}{l}\text { Saneamento in } \\
\text { situ }\end{array}$ & $\begin{array}{l}\text { Tipo de aquífero; Litologia da zona } \\
\text { vadosa; profundidade da zona } \\
\text { subsaturada; qualidade da água. }\end{array}$ & $\begin{array}{l}\text { Vulnerabilidade aquífera e } \\
\text { risco quanto ao } \\
\text { saneamento básico }\end{array}$ \\
\hline $\begin{array}{l}\text { Van Stempvoot } \\
\text { et al., (1993) }\end{array}$ & AVI & $\begin{array}{l}\text { Profundidade da zona subsaturada; } \\
\text { espessura da camada sedimentar; } \\
\text { condutividade hidráulica }\end{array}$ & Vulnerabilidade aquífera \\
\hline Auge (1997) & EKv & $\begin{array}{l}\text { Espessura da zona subsaturada; e } \\
\text { permeabilidade da zona subsaturada }\end{array}$ & Vulnerabilidade aquífera \\
\hline $\begin{array}{l}\text { Doerfliger et al., } \\
\text { (1990) }\end{array}$ & EPIK & $\begin{array}{l}\text { Cobertura protetora; condições de } \\
\text { infiltração; desenvolvimento da rede do } \\
\text { ambiente cárstico e recarga. }\end{array}$ & $\begin{array}{l}\text { Vulnerabilidade aquífera } \\
\text { em meios cársticos }\end{array}$ \\
\hline Auge (2004) & AUGE & $\begin{array}{c}\text { Potencial Hidraulico; Transmissividade } \\
\text { vertical }\end{array}$ & $\begin{array}{c}\text { Vulnerabilidade em } \\
\text { aquíferos } \\
\text { semiconfinados }\end{array}$ \\
\hline $\begin{array}{l}\text { Chachadi; Lobo- } \\
\text { Ferreira (2005) }\end{array}$ & GALDIT & $\begin{array}{c}\text { Tipo de aquífero; condutividade } \\
\text { hidráulica do aquífero; peso da água } \\
\text { subterrânea sobre a água do mar; } \\
\text { Distância para a costa; impacto existente } \\
\text { da intrusão salina; espessura do aquífero } \\
\text { mapeado. }\end{array}$ & $\begin{array}{c}\text { Vulnerabilidade de } \\
\text { aquíferos costeiros } \\
\text { quanto a intrusão salina. }\end{array}$ \\
\hline $\begin{array}{l}\text { Vías et al., } \\
\text { (2006) }\end{array}$ & $\begin{array}{l}\text { Concentration } \\
\text { of flow; } \\
\text { overlying layers; } \\
\text { precipitation - } \\
\text { COP }\end{array}$ & $\begin{array}{l}\text { Concentração do fluxo; camadas } \\
\text { sobrepostas; precipitação pluviométrica. }\end{array}$ & $\begin{array}{l}\text { Vulnerabilidade em } \\
\text { aquíferos cársticos }\end{array}$ \\
\hline $\begin{array}{l}\text { Andrade; Stigter } \\
(2009)^{\star}\end{array}$ & $\begin{array}{l}\text { Multi-method } \\
\text { assessment of } \\
\text { nitrate and } \\
\text { pesticides }\end{array}$ & $\begin{array}{l}\text { Litologia; cobertura de cultivos; } \\
\text { profundidade da zona subsaturada }\end{array}$ & $\begin{array}{l}\text { Vulnerabilidade de } \\
\text { aquíferos aluviais à } \\
\text { contaminação por nitrato } \\
\text { e pesticidas proveniente } \\
\text { do uso agrícola. }\end{array}$ \\
\hline Maia (2011) & MAIA & Profundidade da água; Espessura do & Vulnerabilidade aquífera \\
\hline
\end{tabular}


Conservação e proteção da água subterrânea: uma revisão de metodologias de mapeamento de aquíferos para o ordenamento territorial
Filipe da Silva Peixoto

Itabaraci Nazareno Cavalcante

Karen Vendramini de Araújo Renata Nayara Miranda Câmara Silveira

\begin{tabular}{|c|c|c|c|}
\hline & & $\begin{array}{l}\text { solo; Declividade do terreno; Capacidade } \\
\text { Específica; Recarga Potencial; } \\
\text { Densidade de fraturas; Transmissividade }\end{array}$ & geral \\
\hline $\begin{array}{l}\text { Boi-Roura et al, } \\
(2013)\end{array}$ & $\begin{array}{c}\text { Regression } \\
\text { Model } \\
\text { Assesement of } \\
\text { nitrate }\end{array}$ & $\begin{array}{c}\text { Nitrogênio disponível para incorporação } \\
\text { em água subterrânea; Tipo de aquífero; } \\
\text { presença de solos bem drenados; } \\
\text { percentual de terras irrigadas; indicador } \\
\text { de presença de processo de } \\
\text { desnitrificação } \\
\end{array}$ & $\begin{array}{c}\text { Vulnerabilidade aquífera } \\
\text { geral quanto a } \\
\text { contaminação por nitrato } \\
\text { proveniente de uso } \\
\text { fertilizantes agrícolas }\end{array}$ \\
\hline $\begin{array}{l}\text { Makonto; } \\
\text { Dippenaar, } \\
\text { (2014) }\end{array}$ & $\begin{array}{c}\text { Recharge, } \\
\text { Depth to water } \\
\text { table, Soil } \\
\text { type; Slope - } \\
\text { RDSS } \\
\end{array}$ & $\begin{array}{l}\text { Recarga; profundidade da zona } \\
\text { subsaturada; tipo de solo; condutividade } \\
\text { hidráulica vertical da zona saturada. }\end{array}$ & Vulnerabilidade Aquífera \\
\hline $\begin{array}{l}\text { Lubianetzky et } \\
\text { al., (2015) }\end{array}$ & $\begin{array}{c}\text { Vulnerability } \\
\text { assessment } \\
\text { method }\end{array}$ & $\begin{array}{c}\text { Pluviosidade; topografia; Rochas } \\
\left.\text { fraturadas (transmissividade }-\mathrm{T} /\left[\mathrm{m}^{2} / \mathrm{dia}\right]\right) ; \\
\text { meio poroso c (yrs). }\end{array}$ & $\begin{array}{l}\text { Vulnerabilidade de } \\
\text { aquíferos fissurais ou } \\
\text { fraturados. }\end{array}$ \\
\hline $\begin{array}{c}\text { Kazakis \& } \\
\text { Voudouris }(2015\end{array}$ & $\begin{array}{l}\text { DRASTIC - PA; } \\
\text { DRASTIC - } \\
\text { PAN }\end{array}$ & $\begin{array}{c}\text { Tipo de aquífero; tipo de solo; impacto da } \\
\text { recarga direta na zona vadosa; } \\
\text { profundidade da zona subsaturada; } \\
\text { espessura do aquífero; perdas de } \\
\text { nitrogênio no solo e resistência } \\
\text { hidráulica. } \\
\end{array}$ & $\begin{array}{l}\text { Vulnerabilidade aquífera } \\
\text { geral à contaminação por } \\
\text { nitrato em áreas rurais. }\end{array}$ \\
\hline Oke, et al., 2016 & $\begin{array}{l}\mathrm{RTt}-\text { Rain } \\
\text { Travel time }\end{array}$ & $\begin{array}{l}\text { Quantidade de chuva que infiltra e } \\
\text { percola no solo; o tempo de viagem } \\
\text { (Travel time) é baseado em: } \\
\text { profundidade da zona subsaturada, } \\
\text { condutividade hidráulica de cada } \\
\text { camada; taxa de infiltração; diferença } \\
\text { entre a maior e menor altitude; } \\
\text { porosidade efetiva; número de camadas } \\
\text { superficiais ou subsuperficiais até o topo } \\
\text { do aquífero. }\end{array}$ & $\begin{array}{l}\text { Vulnerabilidade aquífera } \\
\text { para meio intersticial }\end{array}$ \\
\hline Morais (2016) & GATNEK & $\begin{array}{l}\text { Granulometria; Ambiente hidrogeológico; } \\
\text { transmissividade; nível estático; } \\
\text { condutividade hidráulica. }\end{array}$ & $\begin{array}{l}\text { Vulnerabilidade de } \\
\text { aquíferos intersticiais }\end{array}$ \\
\hline
\end{tabular}
Fonte: Elaboração própria.

*nesta metodologia foi utilizado levantamento de parâmetros por meio determinístico estocástico e multivariado neste último, trabalhando com análise fatorial de correspondência.

Modelos não paramétricos baseados em indicadores expressos por interpoladores como krigagem e Inverse Distance Weight - IDW são aplicados para o mapeamento da vulnerabilidade aquífera como foi realizado por Morais (2016), além de metodologias que trabalham a predição de dados para a análise e avaliação de outros modelos. Boy-Roura et al. (2013), por exemplo, desenvolvem um modelo de análise de risco baseado em dados de concentração de nitrato na água subterrânea com a aplicação de interpolação de regressão linear múltipla, cruzando com dados da hidrogeologia local, uso agrícola e ocupação do solo na região de Osona - Espanha.

Também existem diversos modelos desenvolvidos em aplicações específicas com modificações a partir de metodologias preexistentes. O DRASTIC, por ser uma metodologia muito difundida e sendo uma das primeiras a ser desenvolvida, é a mais utilizada nessa situação (JANG et al., 2015; KAZAKIS e VOUDOURIS, 2015). Enquanto Chen et al (2014), Ribeiro (2000) e Antonakos e Lambrakis (2007) desenvolveram um modelo híbrido, incorporando complementos para aplicações mais específicas a partir do DRASTIC.

Modelos matemáticos são pouco utilizados atualmente por conta das incertezas presentes nas diferentes características e parâmetros aquíferos, dificultando a predição dos modelos. Hazic et al. (2015) formularam um modelo conceitual para analisar a diferença básica da quantidade e velocidade da recarga que ocorre nos aquíferos de Sokolovic - Bósnia, e a metodologia foi aplicada de acordo com a diferenciação da equação básica de fluxo da água subterrânea. 


\section{A metodologia DRASTIC}

Dentre as metodologias de vulnerabilidade aquífera mais utilizados, o DRASTIC trabalha com os seguintes parâmetros: Depth to water table; Recharge; Aquifer media; Soil media; Topography; Impact of vadose zone; hydraulic conductivity, baseados em índices a partir de sua integração. Os pesos dos elementos que formam o índice são determinados arbitrariamente a partir de intervalos que variam conforme o parâmetro, e de acordo com Aller et al (1987), os pesos dos parâmetros variam de 1 (menos importante) a 5 (mais importante), enquanto a faixa de classificação varia de 1 à 10 de menos para mais importante.

Os diferentes parâmetros possuem pesos distintos, conforme o grau de influência que estes possuem na vulnerabilidade aquífera (Quadro 2).

Quadro 2 - Peso (ponderação) para os parâmetros.

\begin{tabular}{rr|r}
\hline \multicolumn{2}{c|}{ Parâmetros } & Peso (ponderação) (R) \\
\hline Depth & $\begin{array}{r}\text { Profundidade da zona sub- } \\
\text { saturada }\left(\mathrm{R}_{\mathrm{d}}\right)\end{array}$ & 5 \\
& Recarga (mm/ano) $\left(\mathrm{R}_{\mathrm{r}}\right)$ & 4 \\
\hline Recharge & Meio aquífero $\left(\mathrm{R}_{\mathrm{a}}\right)$ & 3 \\
\hline Soler & Solo $\left(\mathrm{R}_{\mathrm{s}}\right)$ & 2 \\
\hline Topography & Topografia $\left(\mathrm{R}_{\mathrm{t}}\right)$ & 1 \\
\hline Impact & Impacto sobre a zona sub- \\
& saturada $\left(\mathrm{R}_{\mathrm{i}}\right)$ & 5 \\
\hline Conductivity & Condutividade hidráulica \\
& $\left(\mathrm{R}_{\mathrm{c}}\right)$ & 3 \\
\hline
\end{tabular}

Fonte: Aller et al. 1987.

O índice que define a vulnerabilidade aquífera segundo o DRASTIC é definido conforme a Equação 1:

$$
\sum_{i=1}^{7} w i * R i \quad \text { (Equação 1) }
$$

Podendo ser mais expressamente colocada na Equação 2:

$$
I D=D \cdot R d+R e \cdot R r+A \cdot R a+S \cdot R s+T \cdot R t+I \cdot R i+C \cdot R c \quad \text { (Equação 2) }
$$

Onde:

ID = Índice DRASTIC;

$\mathrm{D}=$ profundidade da zona subsaturada, pode ser aferida através da medida do nível estático em poços localizados na área estudada.

$R=$ Recarga que está relativa à quantidade de água que entra no sistema aquífero anualmente o que permite inferir o grau de renovabilidade que a água subterrânea contida no aquífero. A quantificação da recarga em milímetros $(\mathrm{mm})$ pode ser dada por métodos racionais como o balanço hídrico climático a partir da equação de Torntwait e Matter (1955) modificada por Castany (1971), ou por meio empírico de medição direta a partir da oscilação sazonal da zona subsaturada.

$\mathrm{A}=$ Meio aquífero é a caracterização do ambiente hidrogeológico que determina o grau de proteção natural do aquífero. O meio aquífero está relacionado à litologia, bem como o grau de alteração e de fratura das rochas. Isso pode ser definido por mapeamento geológico simples que geralmente já é realizado pelos serviços e órgãos estatais, contudo, é necessário averiguar a compatibilidade entre a escala do mapa e o estudo que se deseja fazer.

$\mathrm{S}=\mathrm{O}$ tipo de solo possui influência na recarga, principalmente, quanto a entrada de contaminantes no meio aquífero e depuração desses contaminantes. Assim, os tipos de solos possuem importância para a definição da vulnerabilidade aquífera.

$\mathrm{T}$ = A topografia está relacionada infiltração da água no solo, e consequentemente à recarga e facilidade de entrada de contaminantes no sistema aquífero. Pode ser mensurada com facilidade por modelos digitais do terreno. 
Conservação e proteção da água subterrânea: uma revisão de metodologias de mapeamento de aquíferos para o ordenamento territorial
Filipe da Silva Peixoto

Itabaraci Nazareno Cavalcante

Karen Vendramini de Araújo Renata Nayara Miranda Câmara Silveira

I = Impacto na zona subsaturada, esse parâmetro busca quantificar o quanto a zona de aeração, ou zona vadosa possui de resistência a passagem da água e consequentemente de contaminantes.

C - Condutividade Hidráulica do meio é uma propriedade que mede a facilidade da movimentação da água no meio (FEITOSA et al., 2008). Deve ser aferida para cada camada com propriedades diferentes conforme a estratificação litológica até o aquífero, e quanto maior o valor, mais rapidamente a água se desloca no meio aquífero.

Os resultados obtidos por meio do índice DRASTIC realçam a diferença entre graus de vulnerabilidade a poluição/contaminação da água subterrânea. Os resultados não são globais, ou seja, não são passíveis de serem comparados de maneira fiel aos dados de outros estudos, mas sim entre as unidades identificadas para um mesmo local. A exemplo disso, Moura et al. (2016) chegam aos seguintes resultados aplicando esse método no município de São Gonçalo do Amarante situado na zona costeira no estado do Ceará - Nordeste do Brasil (Quadro 3):

Quadro 3 - Grau de vulnerabilidade aquífera conforme DRASTIC.

\begin{tabular}{|c|c|c|c|c|c|c|}
\hline$<100$ & $100-119$ & $119-139$ & $120-159$ & $160-179$ & $180-199$ & $>200$ \\
\hline Insignificante & Muito baixa & Baixa & Moderada & Alta & Muito alta & Extrema \\
\hline
\end{tabular}

Fonte: Moura et al., 2016

De modo arbitrário, o pesquisador programa divisões acerca dos limites de graus de vulnerabilidade. Porém esses também podem ser calibrados por análises químicas que comprovem uma efetivação da contaminação da água refletindo as características do uso e ocupação do solo (KASAKIS e VOUDOURIS, 2015; ANTONAKOS e LAMBRAKIS, 2007).

\section{CONCLUSÃO}

A aplicação da vulnerabilidade aquífera é essencial para predição e prevenção da contaminação nas águas subterrâneas. A aplicação precisa ser realizada envolvendo o conceito de vulnerabilidade, que produz subsídios, na qualidade de informações cartográficas, para o planejamento e ordenamento territorial, e para conservação dos recursos hídricos subterrâneos. As metodologias mais utilizadas são baseadas em índices paramétricos. Tanto pela facilidade de informação requente para alimentar a metodologia, como para desenvolver adaptações a partir de metodologias consideradas clássicas, o DRASTIC é vastamente aplicado em diferentes estudos.

As metodologias de vulnerabilidade aquífera tiveram origem no mapeamento de aquíferos, integrando, sobretudo, informações intrínsecas ao meio geológico. Com a evolução dessas metodologias, variáveis mais específicas, como transmissibilidade de meios porosos e fissurais começaram a ser incorporadas, assim como diagnósticos hidrogeológicos de ambientes cársticos associados a precipitação. Outros tipos de vulnerabilidade aquífera foram criados mediante a necessidade de investigação de impacto de determinada atividade ou de determinado contaminante potencial específico, foi o caso de Waste-soil Interaction Matrix quanto aos resíduos industriais, o Brine Disposal Methodology quanto a poços associados a extração de petróleo e o risco por contaminação por hidrocarbonetos. Além do DRASTIC PA; DRASTIC - PAN utilizados para identificar a vulnerabilidade no que se refere ao nitrato gerado pela atividade agrícola.

Os métodos de vulnerabilidade aquífera têm evoluído de gerais para específicos, estes envolvendo determinados tipos de litologias ou ambientes hidrogeológios distintos. Mas, também, é necessário que haja avanço na implementação de modelos que analisam a vulnerabilidade e o risco de maneira combinada, alcançando resultados mais próximos a realidade e permitindo, assim, sua aplicabilidade em ambientes fortemente impactados e em escalas maiores de estudo, como no meio urbano. Além disso, nenhum município brasileiro utiliza a vulnerabilidade aquífera aplicada à política pública ou suporte para leis de uso e ocupação do solo, o que é extremamente recomendado pela Política Nacional de Saneamento Básico e Política nacional de Recursos Hídricos. É necessário, portanto, que haja maior difusão dessas metodologias para que elas estejam mais próximas dos gestores públicos, não somente a nível municipal, mas pensado para o desenvolvimento territorial a nível regional e nacional. 
Conservação e proteção da água subterrânea: uma revisão de metodologias de mapeamento de aquíferos para o ordenamento territorial
Filipe da Silva Peixoto

Itabaraci Nazareno Cavalcante

Karen Vendramini de Araújo Renata Nayara Miranda Câmara Silveira

\section{REFERÊNCIAS}

ADAMS, B.; FOSTER, S. Land-surface zoning for groundwater protection. Journal Institution of Water and Environmental Management, vol. 6, n 4, p. 312-319, 1992. https://doi.org/10.1111/j.17476593.1992.tb00755.x

ALBINET, M.; MARGAT, J. Cartographie de la vulnérabilité à la pollution des nappes d'eau souterraine. Bulletin du BRGM (deuxième série), № 4, p. 13-22, 1970.

ALLER, L., BENNETT, T., LEHR, J.H., PETTY, R., HACKETT, G. DRASTIC: A standardized system for evaluating groundwater pollution in potential using hydrogeologic settings. EPAl600 2- 87035, 1987.

ANDRADE, A.; STIGTER, T. Y. Multi-method assessment of nitrate and pesticide contamination in shallow alluvial groundwater as a function of hydrogeological setting and land use. Agricultural Water Management, Vol. 96, n 12, p. 1751-1765, 2009. https://doi.org/10.1016/.agwat.2009.07.014

ANTONAKOS, A. K.; LAMBRAKIS, N. J. Development and testing of three hybrid methods for the assessment of aquifer vulnerability to nitrates, based on the drastic model, an example from NE Korinthia, Greece. Journal of Hydrology, vol. 333, no 2, p. 288-304. 2007. https://doi.org/10.1016/j.jhydrol.2006.08.014

AUBRE, F. Développement d'une nouvelle méthode d'évaluation de la vulnérabilité des eaux, Paris: département de l'eau, 1992.

AUGE M. P. Investigación hidrogeológica de La Plata y alrededores. Universidad de Buenos Aires. Tesis Doctoral: Buenos Aires, 1997. P. 171

, M. P. Vulnerabilidad de acuíferos. Revista Latino-Americana de Hidrogeologia. Curitiba, 2004, vol. 4, p. 97-103.

BOY-ROURA, M.; NOLAN, B. T.; MENCIÓ, A.; MAS-PLA, J. Regression model for aquifer vulnerability assessment of nitrate pollution in the Osona region (NE Spain). Journal of Hydrology, vol. 505, p. 150162. 2013. https://doi.org/10.1016/j.jhydrol.2013.09.048

CALDWELL, S.; BARRET, K.; CHANG, S. Ranking system for releases of hazardous substance. In: National conference on management of uncontrolled hazardous waste sites, Anais.. Washington D.C. Silver Spring, M.D. Hazardous Material Control Research Institute, p. 14-20. 1981

CARTER, A.; PALMER, R.; MONKHOUSE, R. Mapping the vulnerability of Groundwater to pollution from agriculture practice particularly in respect of nitrate. In: International conference on vulnerability of soil and groundwater to pollutant. Anais. The Hague: TNO Committee on Hydrological Research; Bilthoven: National Institute of Public Health and Environmental Hygiene, Noordwijk, 1987.

CASTANY, G. Tratado Práctico de las Aguas Subterráneas. Ed. Omega, Barcelona, 1971.

CHACHADI, AG, LOBO-FERREIRA, J. P. Assessing aquifer vulnerability to seawater intrusion using GALDIT method: part 2-GALDIT indicator descriptions. IAHS and LNEC, Proc. The fourth inter celtic colloquium on hydrology and management of water resources. Anais. Universidade do Minho, Guimarães, 2005.

CHEN, Z.; NGO, H.H.; GUO, W.; LIM, R.; Wang, X.; O'HALLORAN, K.; LISTOWSKI, A.; CORBY, N.; MIECHEL, C. A comprehensive framework for the assessment of new end uses in recycled water schemes. Science of the Total Environment, vol. 470, p. 44-52. 2014. https://doi.org/10.1016/j.scitotenv.2013.09.061

CIVITA, M. La valutazione della vulnerabilità degli acquiferi all'in inquinamento [Assessment of aquifer vulnerability to contamination]. Proc. 1 st Conv. Naz. Protezione e Gestione delle Acque Sotterranee: Metodologie, Techologie e Obiettivi, Marano sul Parano des Sciences et Génie, 1990.

DOERFLIGER, N.; JEANNIN, P.Y. ZWAHLEN, F. Water vulnerability assessment in karst environments: a new method of defining protection areas using a multi-attribute approach and GIS tools (EPIK method). Environ Geol, vol. 39, p. 165-176, 1990. https://doi.org/10.1007/s002540050446

DUARTE, U. Geologia ambiental da área de São Pedro, SP: vetor águas subterrâneas. Instituto de Geociências, Universidade de São Paulo, São Paulo, 1980. 
Conservação e proteção da água subterrânea: uma revisão de metodologias de mapeamento de aquíferos para o ordenamento territorial
Filipe da Silva Peixoto

Itabaraci Nazareno Cavalcante

Karen Vendramini de Araújo Renata Nayara Miranda Câmara Silveira

ERKAN, P. A New and soft urban planning paradigm: the strategic spatial planning. Debreceni müszaki közlemények, , vol. 2, n 1, p. 89-100. 2008.

ESTABA, R. M. La descentralización y la ordenación del territorio de Venezuela: estratégias hacia la modernidad. Scripta Nova. Revista eletrônica de geografia y ciencias sociales. Barcelona: Universidad de Barcelona, vol. 54, p. 123 - 134. 1999.

EVANS, T. A.; MAIDMENT, D. R. A. Spatial and statistical assessment of the vulnerability of texas groundwater to nitrate contamination. CRWR Online Report, vol. 95, № 2, p. 232, 1995.

FETTER, C. W. Applied hydrogeology. 3o ed. Springer: Berlin, 1994.

FOSTER, S.; HIRATA, R.C.A. Groundwater pollution risk evaluation: the methodology using available data. CEPIS/PAHO/WHO. Lima, 1988.

FOSTER, S; HIRATA, R. C. A. O. Determinação do risco de contaminação das águas subterrâneas: um método baseado em dados existentes. São Paulo: Instituto Geológico, 1993.

FOSTER, S.; HIRATA, R.; GOMES, D.; D'ELIA, M.; PARIS, M. Groundwater: Quality protection. Washington Dc: The World Bank, 2007.

FREEZE, R. A.; CHERRY, J. A. Groundwater. Prentice-Hall International, London, 1987.

GOGU, R.C., DASSARGUES, A. Current trends and future challenges in groundwater vulnerability assessment using overlay and index methods. Environmental Geology, vol. 39, n 6, p. 549-559. 2000. https://doi.org/10.1007/s002540050466

HAERTLE, A. Method of working and employment of EDP during the preparation of groundwater vulnerability maps. Groundwater in Water Resources Planning, n 142, p. 1073-1085, 1983.

HARGERTY, J. D.; PAVONI, J. L.; HEER, J. E. Solid water management. New York, 1973.

HIRATA, R. C. A. Oito perguntas e oito tentativas de respostas sobre a vulnerabilidade à poluição de aquífero. Anais.. In: I Seminario-Taller. Protección de Acuíferos Frente a la Contaminación: metodologia. Toluca, México, 2001.

JANG, C.; LING, C.; LIANG, C.; CHEN, S. Developing a reliable model for aquifer vulnerability. Stochastic Environmental Research and Risk Assessment. vol. 30, n. 1, p. 175-187, 2016. https://doi.org/10.1007/s00477-015-1063-z

JANG, C.; CHEN, S. Integrating indicator-based geostatistical estimation and aquifer vulnerability of nitrate$\mathrm{N}$ for establishing groundwater protection zones. Journal of Hydrology, vol. 523, p. 441-45, 2015. https://doi.org/10.1016/i.jhydrol.2015.01.077

JARVIS, T. W. et al. International borders, groundwater flow and hydroschizophrenia. Ground water, vol. 43, n. 5, p. 764-770. 2005. https://doi.org/10.1111/j.1745-6584.2005.00069.x

KAZAKIS, N.; VOUDOURIS, K. S. Groundwater vulnerability and pollution risk assessment of porous aquifers to nitrate: modifying the DRASTIC method using quantitative parameters. Journal of Hydrology, vol. 525, p. 13-25, 2015. https://doi.org/10.1016/j.jhydrol.2015.03.035

KULFS, C. Rating the Hazard potential on waste disposal facilities. In: National conference on management of uncontrolled hazardous waste sites, Anais.. Washington D.C. Silver Spring, M.D. Hazardous Material Control Research Institute, p. 30-41. 1980

KUMAR, P. Bansod, B. K. S.; Debnathe, S. K.; Thakur, P. .; Granshuamt, C. Index-based groundwater vulnerability mapping models using hydrogeological settings: A critical evaluation. Environmental Impact Assessment Review, 2015. Vol. 51, p. 38-49. https://doi.org/10.1016/j.eiar.2015.02.001

LE GRAND, H. E. A. standardized system for evaluating waste disposal sites: a manual to accompany description and rating charts. Worthington, $\mathrm{OH}$ : National Water Well Association, 1983. https://doi.org/10.1002/j.1551-8833.1964.tb01292.x

LE GRAND, H. System for evaluating contamination potential of some waste disposal sites. Journal American Water Work Association, vol. 56, no 8, p. 959-974. 1964. 
Conservação e proteção da água subterrânea: uma revisão de metodologias de mapeamento de aquíferos para o ordenamento territorial
Filipe da Silva Peixoto

Itabaraci Nazareno Cavalcante

Karen Vendramini de Araújo

Renata Nayara Miranda Câmara Silveira

LUBIANETZKY, T. A.; DICKSON, S. E.; GUO, Y. Proposed method: incorporation of fractured rock in aquifer vulnerability assessments. Environmental Earth Sciences, vol. 74, oㅜ 6, p. 4813-4825, 2015. https://doi.org/10.1007/s12665-015-4471-y

MAIA, P. H. P. Um novo método para avaliar a vulnerabilidade dos aquíferos. Instituto de Geociências, Tese de doutoramento, Universidade Federal da Bahia, Salvador, 2011.

MAKONTO, O. T.; DIPPENAAR, M. A. Aquifer vulnerability using recharge, depth to groundwater, soil type and slope to classify the vadose zone (Molototsi and Middle Letaba quaternary catchments, Limpopo Province, South Africa). Environmental Earth Sciences, vol. 72, n. 5, p. 1615-1623, 2014. https://doi.org/10.1007/s12665-014-3065-4

MARCOLONGO, B.; PRETTO, L. Vulnerabilità degli acquiferi nella pianura a nord di Vicenza. Padova: Grafiche Erredici. 13 p. (Publicación CNR-GNDCI-CNR n. 28), 1987.

MONKHOUSE, R. A. Vulnerability of aquifers and groundwater quality in the United Kingdom. Nottingham, Reino Unido: Institute of Geological Sciences Report, 1983.

MORAG, N. Water, Geopolitics and State Building: The Case of Israel. Middle Eastern Studies, vol. 37, n. 3, p. 179-198, 2010. https://doi.org/10.1080/714004411

MORAIS, J. B. A. Vulnertabilidade e risco à poluição: contaminação das águas subterrâneas na zona portuária do Pecém. Tese de doutoramento, Universidade Federal de Pernambuco, Recife, 2016.

MOURA, P.; SABADIA, J. A. B.; CAVALCANTE, I.N. Mapeamento de Vulnerabilidade dos Aquíferos Dunas, Barreiras e Fissural na Porção Norte do Complexo Industrial e Portuário do Pecém, Estado do Ceará. Geociências (São Paulo. Online), vol. 35, p. 77-89, 2016.

MUHAMMAD, A. M. ZHONGUAN, T.; DAWOOD, A. S.; EALR, B. et al. Evaluation of local groundwater vulnerability based on DRASTIC index method in Lahore, Pakistan. Geofísic Internacional, vol. 1, n. 54, p. 67-81. 2015. https://doi.org/10.1016/i.gi.2015.04.003

MURAT, V. Étude comparative des méthodes d'évaluation de la vulnerabilité intrin'seque des aquifères à la polluition application aux aquifères granulaires du Piémont Laurentien. Thèse du doctorat, Université Du Québec, Quebéc, 2000.

PEIXOTO, F. S. SILVEIRA, R. N. C. M. Watershed: trends and approach of applicability in urban environment. Revista Brasileira de Geografia Física, vol. 10, ํㅡ 3, p. 767-783. 2017. https://doi.org/10.5935/1984-2295.20170054

PHILIPS, C.; NATHWANI, J.; MOOIJ, H. Development of a soil-waste interaction matrix for assessing land disposal of industrial wastes. Water Resources Research, Washington, D.C. vol. 11, p. 859-868, 1977. https://doi.org/10.1016/0043-1354(77)90073-2

RAO, P. S. C.; HORNSBY, A. G.; JESSUP, R. E. Indices for ranking the potential for pesticide contamination of groundwater. Soil and Crop Science Society of Florida proceedings, vol. 44, p. 1-8. 1985.

REBOUÇAS, A. C. Água na Região Nordeste: desperdício e escassez. Estudos Avançados, São Paulo, vol. 29, no 11, p. 127-154, 1997. https://doi.org/10.1590/S0103-40141997000100007

REZENDE, D. A. Planejamento estratégico municipal como proposta de desenvolvimento local e regional de um município paraense. Ver. FAE, Curitiba,, vol. 9, n. 2, p. 87 - 104, 2016.

ROGERS, P. P. Water governance, water security and water sustainability. In: Rogers, P. P. et al. (Ed.) Water crisis: myth or reality? London: Fundación Marcelino Botín, Taylor \& Francis, p. 3-36. 2006. https://doi.org/10.1201/9781439834275.pt1

SILVA, C.; AMIR, Y.; PENNA, Y. Controle da poluição das águas subterrâneas do Vale do Paraíba. Anais.. In: Congresso brasileiro de águas subterrâneas, 1. Recife, 1980.

SWAIN, A. Challenges for water sharing in the Nile basin: changing geo-politics and changing climate. Hydrological Sciences Journal, vol. 56, n. 4, p. 687-702, 2011. https://doi.org/10.1080/02626667.2011.577037

TALTASSE, P. Mapas de vulnerabilidade à poluição dos lençóis aquíferos do município de Campinas (SP). 1. Ed, São Paulo: Editora USP / Instituto de Geociências, 1972. 
UNITED STATES EVIRONMENT PROTECTION AGENCY - EPA. A review of methods for assessing aquifer sensitivity and groundwater vulnerability to pesticide contamination. EPA, office of water (4602), 813-R-93-002, 147, 1993.

VİAS, J.M. B. ANDREO, J.M. PERLES, F. CARRASCO, I. VADILLO. Proposed method for groundwater vulnerability mapping in carbonate (karstic) aquifers: the COP method. Hydrogeol, vol. 14, n. 6, p. 912925, 2006. https://doi.org/10.1007/s10040-006-0023-6

VILLAÇA, F. Dilemas do plano diretor. In: 0 município no século XXI: cenários e perspectivas. Ed. especial. São Paulo: Fundação Prefeito Faria Lima - CEPAM, 1999.

VILLAR, P. C. Groundwater and the Right to Water in a Context of Crisis. Ambiente \& Sociedade, vol. 19, n. 1, p. 85-102, 2016. https://doi.org/10.1590/1809-4422asoc150126r1v1912016

VINUTU, J. A amostragem em bola de neve na pesquisa qualitativa: um debate em aberto. Temáticas, Campinas, vol. 22, n. 44, p. 203-220, 2014.

VRBA, J. E. ZAPOROZEC, A. Guidebook on mapping groundwater vulnerability. Alli, 1994.

Recebido em: 19/05/2018

Aceito para publicação em: 09/04/2020 